\title{
Efficacy of School-Based Interventions for Improving Muscular Fitness Outcomes in Adolescent Boys: A Systematic Review and Meta-analysis
}

\author{
Ashley Cox ${ }^{1} \cdot$ Stuart J. Fairclough ${ }^{1} \cdot$ Maria-Christina Kosteli $^{1} \cdot$ Robert J. Noonan ${ }^{2}$
}

Published online: 15 November 2019

(c) The Author(s) 2019

\begin{abstract}
Background It has been reported that boys' and girls' physical activity (PA) levels decline throughout adolescence. Boys are at risk of physical inactivity during adolescence; however, in intervention research, they are an under-represented group relative to girls. It is suggested that the school environment may be central to developing interventions that support adolescents in meeting the current PA guidelines. The aim of this systematic review and meta-analysis was to investigate the efficacy of school-based physical activity interventions for improving muscular fitness (MF) in adolescent males.

Methods This systematic review and meta-analysis followed the preferred reporting systems for meta-analyses guidelines and was registered on PROSPERO (Registration number: CRD42018091023). Eligible studies were published in English within peer-reviewed articles. Searches were conducted in three databases, with an additional grey literature search in Google Scholar. Studies investigating MF outcomes were included.

Results There were 43 data sets identified across 11 studies, from seven countries. Overall methodological quality of the studies was moderate-to-strong. Interventions targeting MF evidenced a small-to-medium effect $(g=0.32$, CI $0.17,0.48$, $p<0.00)$. Subgroup analyses of MF delivery method resulted in small-to-medium effects: upper limb MF measures $(g=0.28$, 95\% CI $-0.02,0.58, p=0.07)$, lower limb MF measures $(g=0.28,95 \%$ CI 0.09, 0.68, $p=0.03)$, combined MF activities ( $g=0.24,95 \% \mathrm{CI}-0.04$ to $0.49, p=0.05)$, plyometric activities $(g=0.39,95 \% \mathrm{CI} 0.09,0.68, p=0.01)$, body weight $(g=0.27$, $95 \%$ CI $-0.10,0.65, p=0.15)$, and traditional MF methods ( $g=0.43,95 \%$ CI $0.09,0.78, p=0.01)$.

Conclusions School-based interventions which aimed to increase MF outcomes in adolescent boys demonstrated smallto-moderate effects. Traditional and plyometric methods of resistance training appear to be the most effective form of PA delivery in adolescent males. More quality research is required to assess the impact of MF delivered in the school environment to inform future intervention design.
\end{abstract}

\section{Introduction}

It is recommended that adolescents engage in a minimum of 60 min of moderate-to-vigorous physical activity (MVPA) per day with muscle and bone strengthening exercise

Electronic supplementary material The online version of this article (https://doi.org/10.1007/s40279-019-01215-5) contains supplementary material, which is available to authorized users.

Ashley Cox

23730170@edgehill.ac.uk

1 Movement Behaviours, Health and Wellbeing Research Group, Department of Sport and Physical Activity, Edge Hill University, Ormskirk, UK

2 Appetite and Obesity Research Group, Department of Psychological Sciences, University of Liverpool, Liverpool, UK

\section{Key Points}

MF interventions delivered in a school-based environment demonstrated small-to-moderate effects in adolescent boys.

MF delivered in a traditional manner, such as weight machines and free weights, may have a greater effect on enhancing MF than other forms of MF delivery.

Plyometric forms of MF delivery demonstrated significant homogeneous effects and require further quality research to assess their application in the school environment. 
(MBSE) to be incorporated three times per week [1-4]. A recent systematic review confirmed the associated health benefits of meeting the recommended MVPA guideline [5]. Furthermore, participating in the recommended 3 days of MBSE per week has also been associated with positive physical and mental health benefits in children and young people [6-10]. Despite this evidence, less than 50\% of young people in Europe meet the recommended amount of MVPA suggested by the World Health Organisation (WHO), with this figure declining with age [11]. There is also an international downward temporal trend in muscular fitness among school children, indicating a lack of activities that support the development of muscular fitness [12-15]. Muscular fitness is assessed by measuring performance in tests of muscular strength, power, and muscular endurance [12], and forms part of the MBSE guideline for PA. Lower levels of muscular fitness are associated with the development of noncommunicable disease in adolescent populations [16-21]. Moreover, the development of muscular fitness has been correlated with enhanced bone health, enhanced motor skill, and decreased fat mass in adolescents [22-24].

The benefits of MBSE are well established, supported by position stands from leading organisations [25, 26]. Despite the growing body of literature supporting the benefits of $\mathrm{MF}$, it is often the overlooked element of PA guidelines. Recent UK estimates for health care costs associated with muscle weakness, defined by low grip strength according to the Foundation for the National Institutes of Health criteria (men $<26 \mathrm{~kg}$, women $<16 \mathrm{~kg}$ ), exceed £2.5 billion [27]. Furthermore, the United States reported estimated health care costs associated with muscular weakness at $\$ 18.5$ billion [28]. Poor muscular fitness is associated with sarcopenia, poor quality of life, loss of functional movement, and increasing the likelihood of contracting a non-communicable disease [29]. The associated health care costs and accompanying pathologies support the need to address the downward trend in muscular fitness currently witnessed in youth.

The school environment has been shown to be effective in the promotion of PA in adolescents [30]. Adolescents are most active during the school day compared to evenings and weekends [31]. Additionally, the school environment provides access to PA independent of background or socioeconomic status [32]. This may expose adolescents to varying forms of PA that they may not have been exposed to outside of school. However, the efficacy of school-based interventions investigating PA in adolescent males is unclear. Much of the existing research and policy to promote PA is directed towards adolescent girls, suggesting that males are at low risk of not meeting the suggested PA levels indicative of good health [33-36]. However, boys are reported to be at greater risk than girls of becoming overweight or obese, compromising short- and long-term health [36-40]. Recent national surveillance data suggest that adult males may be more likely to be overweight when compared to adult females [41, 42]. Additionally, worldwide trends in BMI are increasing year on year, with Asia displaying a period of acceleration [43]. For male adolescents, healthy behaviours catalysed during adolescence are often carried into adulthood, supporting the need to investigate the efficacy of current interventions [44].

It is hypothesised that male adolescents may respond more favourably towards resistance training (RT) as these activities are perceived as masculine $[45,46]$. Furthermore, existing evidence supports the role of MF interventions for improving physiological and psychological health $[6,8,47]$. However, research suggests that the development of MF in upper and lower limbs is not homogeneous, and may vary throughout growth and maturation [48-50]. The heterogeneous nature of MF development in adolescent boys may not be accounted for when prescribing RT on a large scale. Understanding how this phenomenon impacts school-based delivery of RT may support future intervention design when attempting to cater for multiple participants. Additionally, appropriate forms of RT delivery may engage overweight or obese adolescents [51]. Implementing effective RT interventions in the school environment may allow overweight and obese youth to excel by taking advantage of their relatively greater absolute strength [51]. Therefore, RT may be a way of increasing PA levels and improving health among overweight or obese adolescents. However, RT is often an overlooked element of PA guidelines when considering the development of school-based interventions and requires contextualisation.

When exploring the existing literature that reports on the efficacy of MVPA interventions across both sexes and age ranges, mixed outcomes have been reported with small changes of around 4 min per day following school-based interventions [52]. However, it is unclear how adolescent boys respond to school-based RT interventions. To the authors' knowledge, this review is the first to investigate the efficacy of school-based PA interventions to improve MF outcomes in adolescent boys. This systematic review and meta-analysis will include studies that (1) represent adolescent boys and report MF outcomes; and (2) determine the efficacy of RT interventions delivered in school settings. Thus, the purpose of this systematic review and meta-analysis is to investigate the efficacy of school-based interventions on MF outcomes in adolescent boys.

\section{Methods}

\subsection{Protocol and Registration}

This systematic review and meta-analysis were registered with PROSPERO on 15th March, 2018 (Registration 
number: CRD42018091023). The protocol is published online (https://www.crd.york.ac.uk/prospero/display_recor d.php?RecordID=91023), and follows the PRISMA statement for reporting systematic reviews and meta-analyses.

\subsection{Search Procedure}

A systematic search was conducted in April 2018 using three electronic databases (PubMed, SPORT Discus, and Web of Science). A grey literature search of Google Scholar was also conducted to minimise publication bias [53]. Journal articles published in English post-May 2010 until the date of the final search in August 2018 were considered for review. May 2010 was chosen as the initial reference point to capture all interventions conducted, following the publication of the WHO PA guidelines [1]. WHO guidelines were used as the PA guideline reference to provide a balanced search strategy, accounting for all countries, including those yet to establish their own PA policy and guidelines [54]. The search strategies for each database are detailed in Table S1 as supplementary information, with a link to one of the database searches as per PRISMA guidelines. The PRISMA flow diagram detailing the procedure can be found in Fig. 1.
Reference lists of relevant articles, including systematic literature reviews, were examined for potential articles which fitted the criteria. A recent systematic review that reflects the target population group and training intervention for this review was also checked for any further literature [6]. All search results were exported to a reference manager, Covidence (https://www.covidence.org; Covidence, Melbourne, Australia), allowing for central reviewing and collection of all texts for screening.

\subsection{Study Selection}

Studies were eligible if they contained an intervention where the main purpose was to promote PA in the school environment, with the primary outcome of increasing objectively measured MF. Included studies investigated adolescent boys aged 10-18 years. Mixed boys' and girls' data were acceptable if sex-specific results were available and/or accessible. Studies must have been conducted in a school or college between 8 am- 6 pm on week days during term time. Studies were included if MF measures were taken at baseline and at the end of the intervention. Girls, community interventions, elite sport, and thesis/dissertations were excluded. Measures
Fig. 1 PRISMA flow diagram to show each stage of the systemic eligibility process
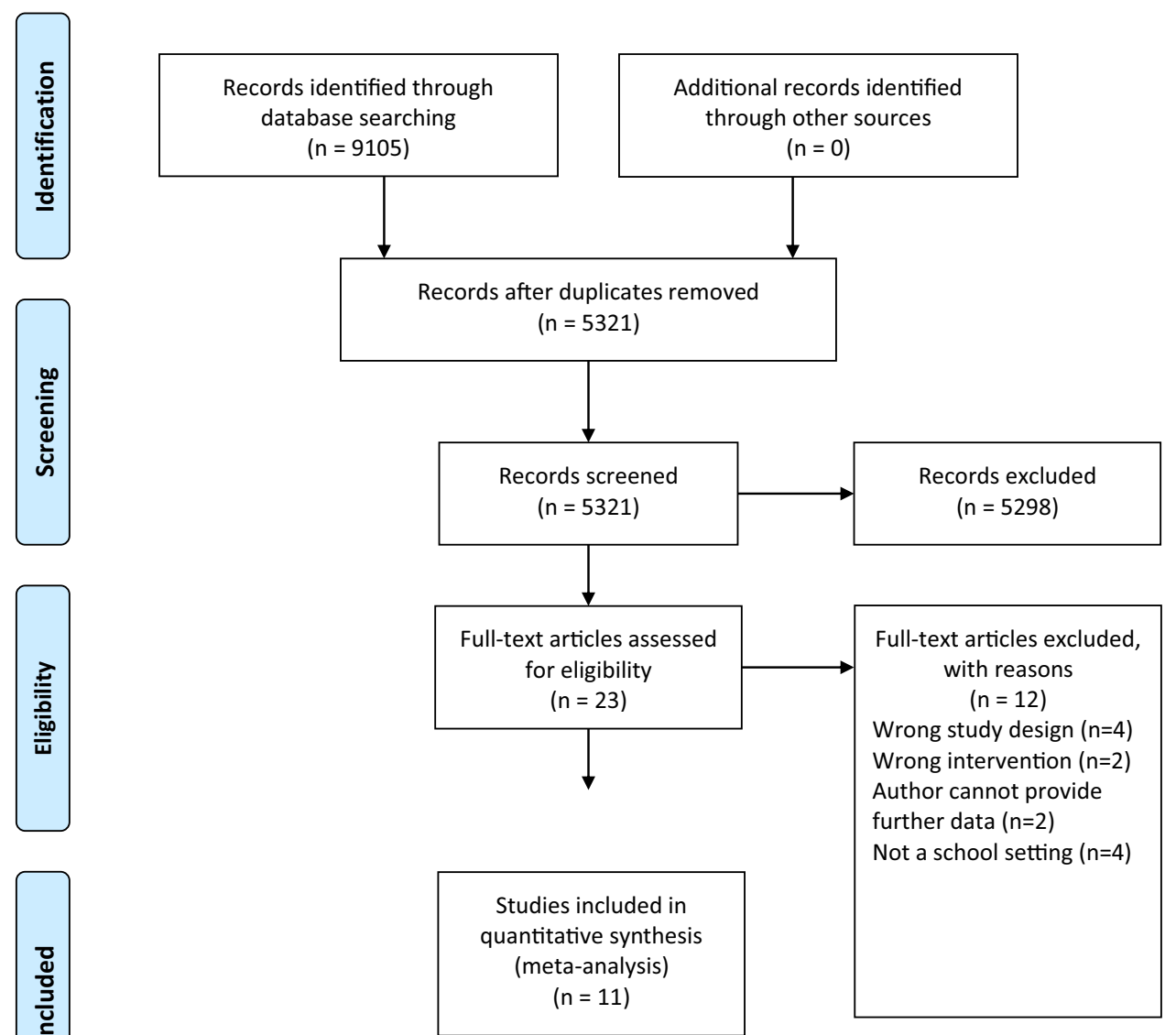
of MF had to have been documented in their use previously in peer-reviewed research and could not be novel or firsttime iterations of a testing protocol.

Studies could be randomised or non-randomised. Research studies published before 2010 were excluded as were studies that were not published in English. Where full texts were not readily available and where only partial data were reported, the study authors were contacted and asked to provide the full-text version with the accompanying data in full. If no response was received after an 8-week follow-up reminder, these studies were excluded as they could not be fully assessed for eligibility. A total of 11 authors were contacted to provide further data and full texts. From the authors contacted, five non-responses were recorded, with a further two authors unable to provide further data for analysis.

\subsection{Data Extraction and Risk of Bias}

All search results were exported into Covidence (https:// www.covidence.org; Covidence, Melbourne, Australia) and duplicates were removed. The first author (AC) screened all titles and abstracts for obvious irrelevance, $10 \%$ were also checked by another author (RN). The $10 \%$ screening figure is a recognised validation and agreement threshold for systematic reviews [55]. The full text of eligible studies was then located and reviewed by two authors (AC and RN). Any disagreements were resolved in a meeting involving three authors (AC, RN, and SF). Study data were extracted by $\mathrm{AC}$ and included study characteristics (i.e., country, year); participant characteristics (e.g. sample size, age, anthropometrics); intervention components (i.e., setting, duration, intervention); and changes in the outcomes (i.e., change in grip strength). The outcome data were extracted in the form of mean, standard deviation, and sample size. Included studies were assessed for risk of bias using a modified tool $[56,57]$ appropriate for PA reviews which included measures for quantitative studies.

\subsection{Data Synthesis and Analysis}

Random effects meta-analyses were conducted using Comprehensive Meta-analysis Software (Version 2.2.064). Raw scores were converted to standardised means data. Studies that reported more than one measure of assessing a single outcome (i.e., vertical jump height and reactive strength index for lower limb outcome) were converted into a single common effect size for the analysis to avoid inflating sample sizes. A random effects model was considered more appropriate for this review to account for the expected heterogeneity between PA measures [58]. Hedges' $g$ with 95\% CIs were used to calculate effect sizes [59]. Pooled weighted standard deviations were used as per the Hedge's $g$ formula and based on a positive effect direction [59]. Hedges' $g$ was interpreted using Cohen's [59] effect sizes, as small (0.2), medium (0.5), and large (0.8). Heterogeneity was assessed using $I^{2}$ statistic, with values of 25,50 , and 75 representing low, medium, and high heterogeneity, respectively [60]. Publication bias was assessed using Egger's statistic, where bias was deemed to be present at $p<0.05$ [61]. Corresponding funnel plots were created for visual interpretation, followed by calculating Egger's statistic to confirm or refute publication bias.

\subsection{Quality Appraisal}

Included studies were assessed for risk of bias using a modified tool suitable for PA interventions that included nonRCT designs [56, 57]. The ability to distinguish the nature of the PA outcome assessment method in addition to the

Table 1 Quality assessment (risk of bias)

\begin{tabular}{|c|c|c|c|c|c|}
\hline Study & $\begin{array}{l}\text { Appropriate sequence } \\
\text { generation and/or } \\
\text { randomisation }\end{array}$ & $\begin{array}{l}\text { Allocation con- } \\
\text { cealment and/or } \\
\text { blinding }\end{array}$ & $\begin{array}{l}\text { Complete outcome data and/ } \\
\text { or low withdrawal/dropout } \\
(<20 \%)\end{array}$ & $\begin{array}{l}\text { Appropriate } \\
\text { outcome meas- } \\
\text { ure (PA) }\end{array}$ & $\begin{array}{l}\text { Quality } \\
\text { score }\end{array}$ \\
\hline 1. De Souza et al. (2015) [62] & & & $\mathrm{X}$ & $\mathrm{X}$ & 2 \\
\hline 2. Eather et al. (2016) [63] & $\mathrm{X}$ & $\mathrm{X}$ & $\mathrm{X}$ & $\mathrm{X}$ & 4 \\
\hline 3. Giannaki et al. (2016) [64] & & $\mathrm{X}$ & $\mathrm{X}$ & $\mathrm{X}$ & 3 \\
\hline 4. Kennedy et al. (2018) [65] & $\mathrm{X}$ & & $\mathrm{X}$ & $\mathrm{X}$ & 3 \\
\hline 5. Lloyd et al. (2012) [66] & & & $\mathrm{X}$ & $\mathrm{X}$ & 2 \\
\hline 6. Lloyd et al. (2016) [67] & & & $\mathrm{X}$ & $\mathrm{X}$ & 2 \\
\hline 7. Lubans et al. (2016) [68] & $\mathrm{X}$ & $\mathrm{X}$ & & $\mathrm{X}$ & 3 \\
\hline 8. Muehlbauer et al. (2012) [69] & & & $\mathrm{X}$ & $\mathrm{X}$ & 2 \\
\hline 9. Muntaner-mass and Palou (2017) [70] & & & $\mathrm{X}$ & $\mathrm{X}$ & 2 \\
\hline 10. Weeks and Beck (2012) [71] & & & $\mathrm{X}$ & $\mathrm{X}$ & 2 \\
\hline 11. Winwood and Buckley (2017) [72] & & & $\mathrm{X}$ & $\mathrm{X}$ & 2 \\
\hline
\end{tabular}

$\mathrm{X}=$ the study demonstrated appropriate steps to account for the respective risk of bias confounder 
existent randomisation, blinding, and complete outcome data items was accounted for within this tool. This adapted quality assessment tool used a 1-4 scoring system (i.e., $1=$ weak and $4=$ very strong; see Table 1 .

\section{Results}

Extracted studies were conducted in seven countries (UK, Brazil, Australia, Cyprus, Germany, Spain, and New Zealand) [62-72]. The studies included displayed no obvious bias, but rather a lack of depth and detail, which made the risks of bias difficult to detect. Details regarding sequence generation and allocation concealment and/or blinding were found to be the categories that were often not sufficiently reported on. Twenty-seven percent of the studies reported an appropriate sequence generation or randomisation in detail $[63,65,69]$, with a further $27 \%$ reporting allocation concealment or blinding in detail $[63,69,72]$. This may suggest selection and reporting bias in the literature. Complete outcome data and/or low dropout rates were present in $81 \%$ of the included studies and can, therefore, be interpreted as having low risk of bias as a result of attrition. Risk of bias through inappropriate outcome measures was not an issue for this review as all studies selected had to demonstrate an objective way of assessing MF.

Forty-three data sets were extracted from 11 studies [62-72] assessing MF, with studies reporting multiple MF outcomes including a combination of upper and lower limb measures. Upper and lower limb data sets were analysed independently to identify possible intervention effects, categorised by testing site. Further subgroup analyses of MF interventions were conducted, accounting for: bodyweight movements (i.e., push-ups and curl ups), combined activities (i.e., the use of multiple forms of resistance exercise such as bodyweight and plyometric within the same intervention), plyometrics, and traditional methods such as weight machines and free weights. Plyometric training studies had to exclusively state that the intervention utilised the stretchshortening cycle to take advantage of the elastic properties of the muscle to produce power [73, 74]. Participants' ages ranged from 11.0-16.9 years, samples were separated into, MF control $(n=1164)$ and MF intervention $(n=1252)$. A full breakdown of how sample sizes were extracted is provided in Table 2. Identification of possible publication bias was plotted against standard errors to generate funnel plot (Fig. 2). Egger's analyses for all data sets suggested that publication bias was not present $(p>0.05)$.

\subsection{Pooled Analysis: Muscular Fitness}

Muscular fitness interventions demonstrated an overall small-to-medium effect ( $g=0.32$, CI $0.17,0.48, p<0.00$ ).
Medium-to-high heterogeneity was present amongst the 43 data sets $\left(I^{2}=71.50\right)$. The 43 data sets came from 11 studies accounting for different MF outcomes and measures within each intervention and can be seen in Table 2. The overall effect of all interventions investigating MF can be seen in Fig. 3.

\subsection{Upper and Lower Limb Activities}

Muscular fitness outcomes were separated into those that assessed upper limb $(n=14)$ and lower limb muscle outcomes $(n=27)$. Two data sets measuring core strength were omitted from the analysis as this number was insufficient. Upper limb outcomes presented a small-to-medium effect, with moderate heterogeneity $(g=0.28,95 \% \mathrm{CI}-0.02,0.58$, $p=0.07, I^{2}=83.86$ ). Lower limb outcomes displayed less heterogeneity when compared to upper limb $\left(I^{2}=46.41\right)$ and elicited a small-to-medium effect $(g=0.28,95 \%$ CI 0.09 , $0.68, p=0.03$ ). The corresponding forest plot can be seen in Fig. 4.

\subsection{Combined Activities}

Combined activities (CA) consisted of those interventions that incorporated multiple methods to enhance MF, such as plyometric, bodyweight, and traditional methods conducted within the same session $(n=22)$. There was a small effect for these interventions ( $g=0.24,95 \%$ CI -0.04 to 0.49 , $p=0.05)$, which had high heterogeneity $\left(I^{2}=84.86\right)$.

\subsection{Plyometric Activities}

Plyometric forms of training $(n=6)$ resulted in a small-tomoderate effect size $(g=0.39,95 \%$ CI $0.09,0.68, p=0.01)$. Analysis of heterogeneity demonstrated that plyometric forms of training were homogeneous $\left(I^{2}=0.00\right)$.

\subsection{Body Weight Activities}

Interventions utilising body weight (BW) as the resistance elicited a small effect $(n=8, g=0.27,95 \%$ CI $-0.10,0.65$, $p=0.15$ ). Analysis demonstrated medium heterogeneity $\left(I^{2}=51.53\right)$ for all studies utilising BW.

\subsection{Traditional Methods}

Traditional methods (TM) were deemed to be those methods that utilised free weights and resistance machines [75]. TM indicated a small-to-medium effect $(n=7, g=0.43,95 \%$ CI $0.09,0.78, p=0.01$ ). TM displayed low heterogeneity $\left(I^{2}=0.00\right)$ and the greatest effect size in relation to the control groups. The entire breakdown of MF subgroups is presented in Fig. 3. 


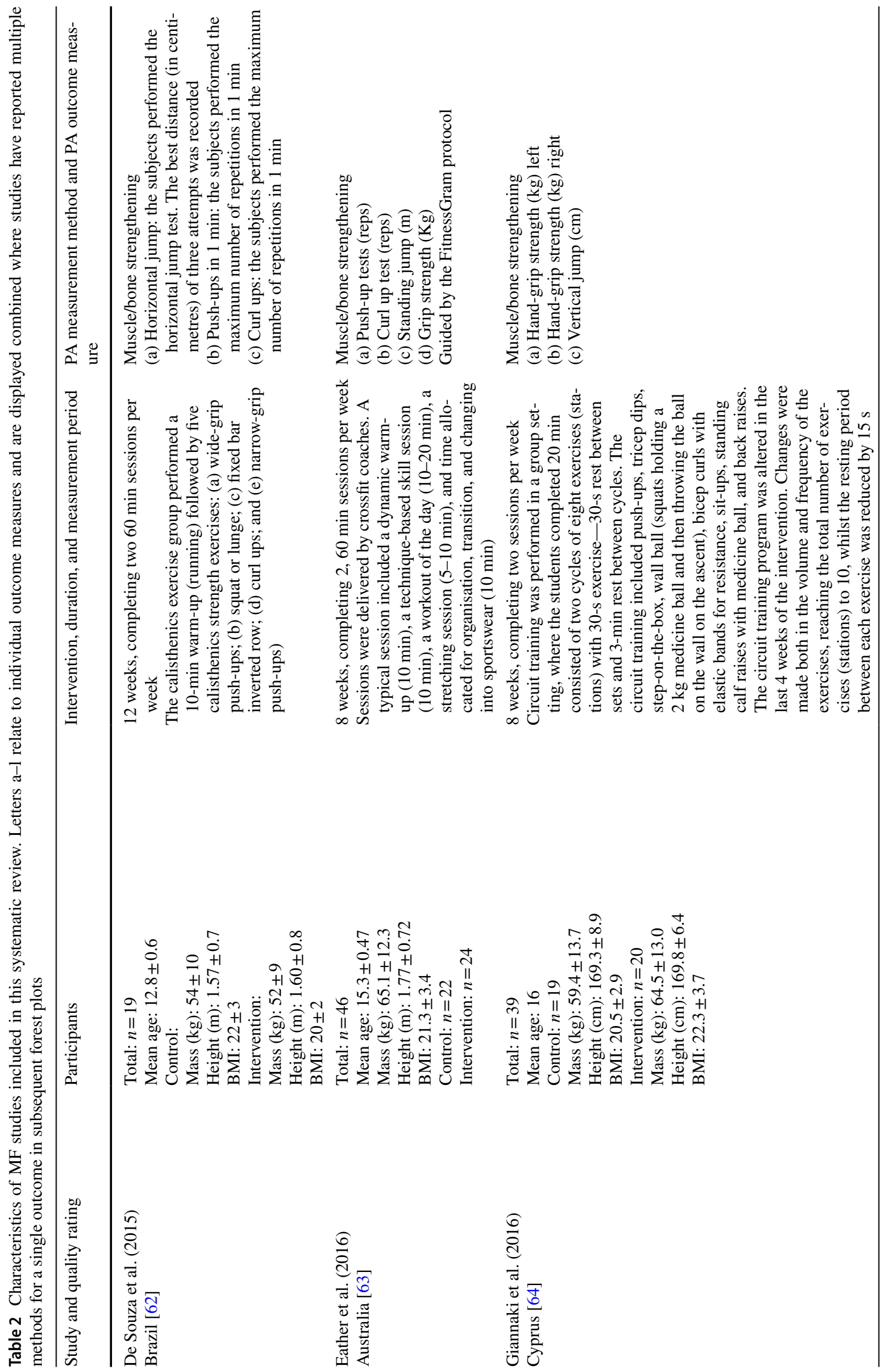




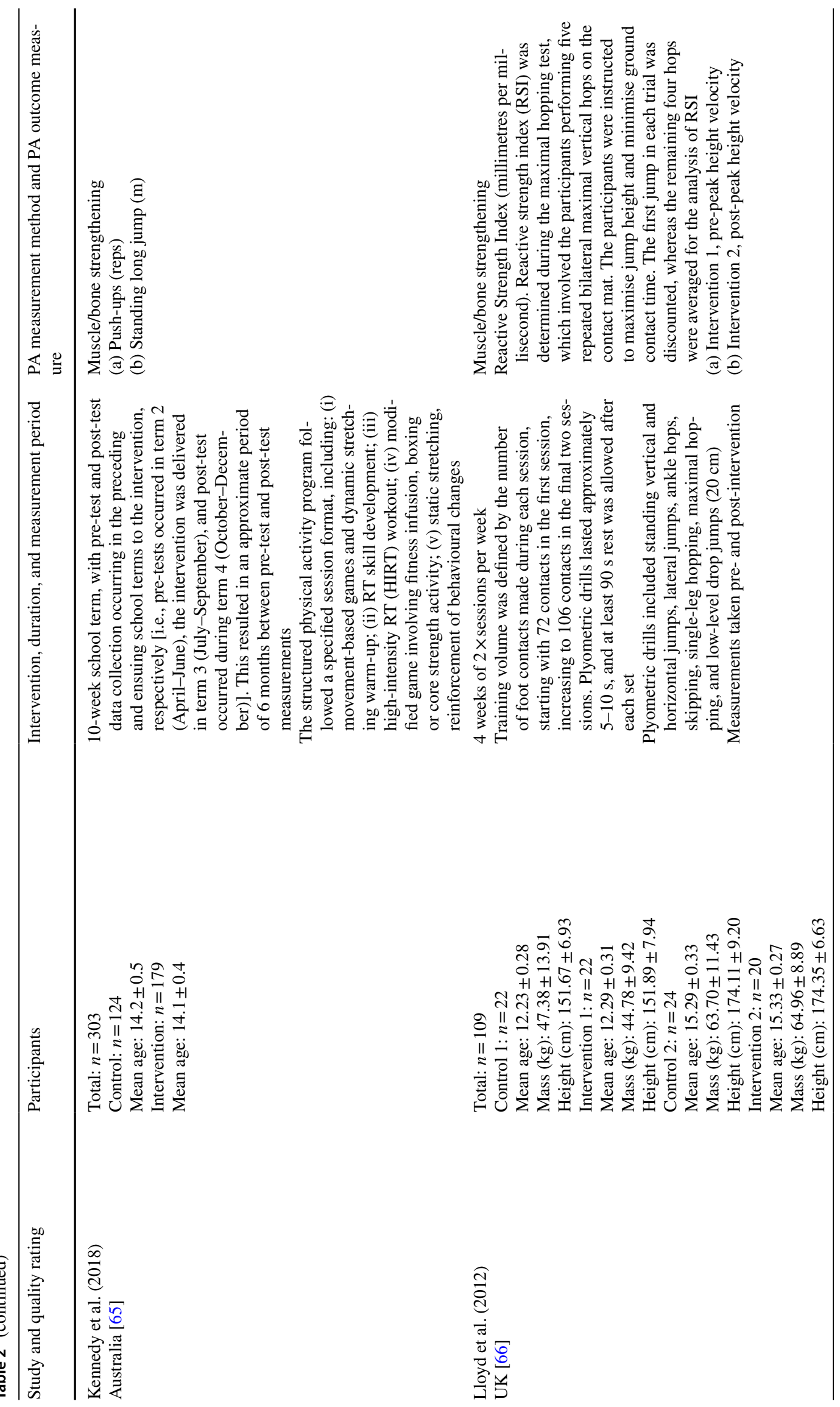




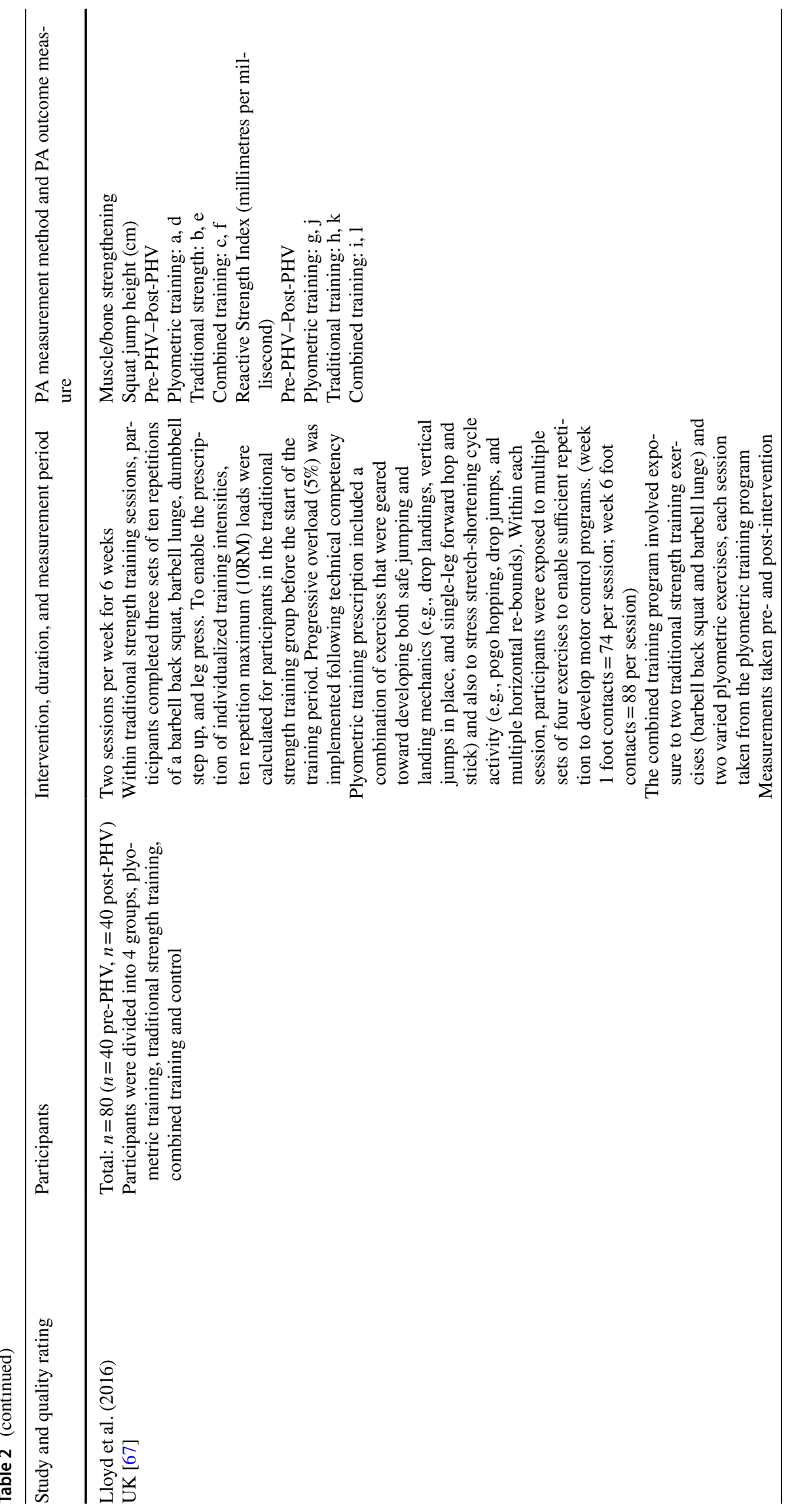




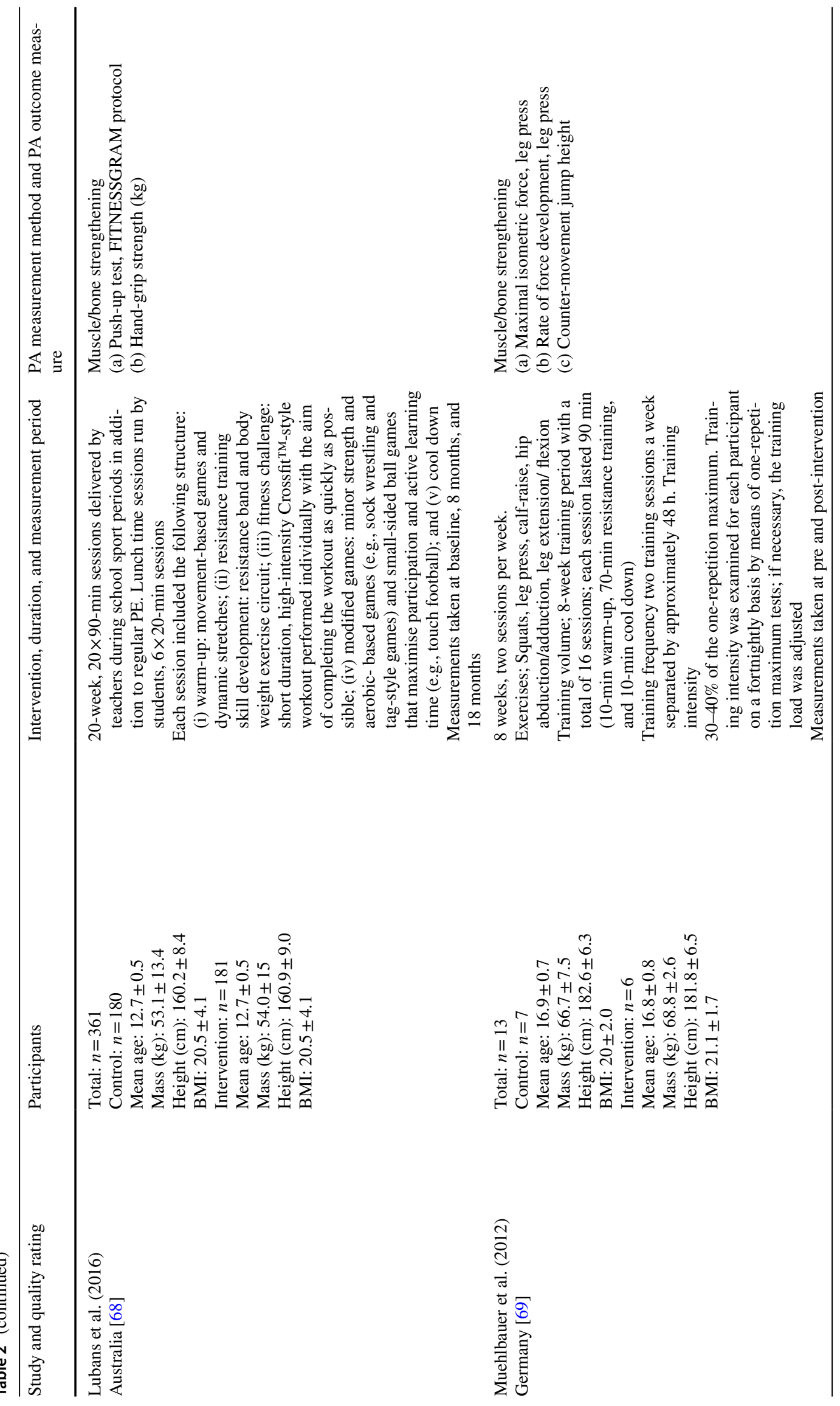




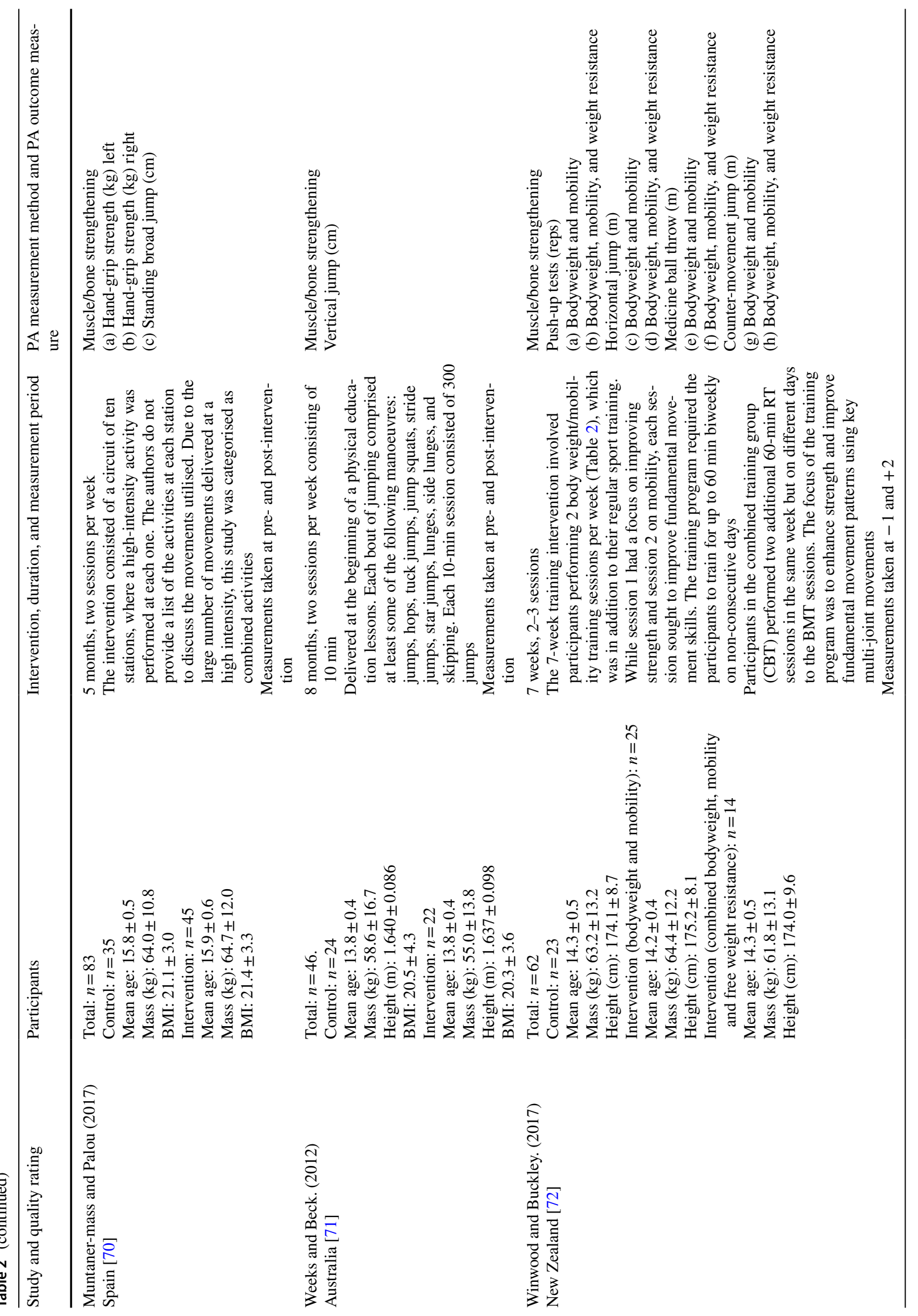


Fig. 2 Funnel plot of standard error by Hedge's $g$

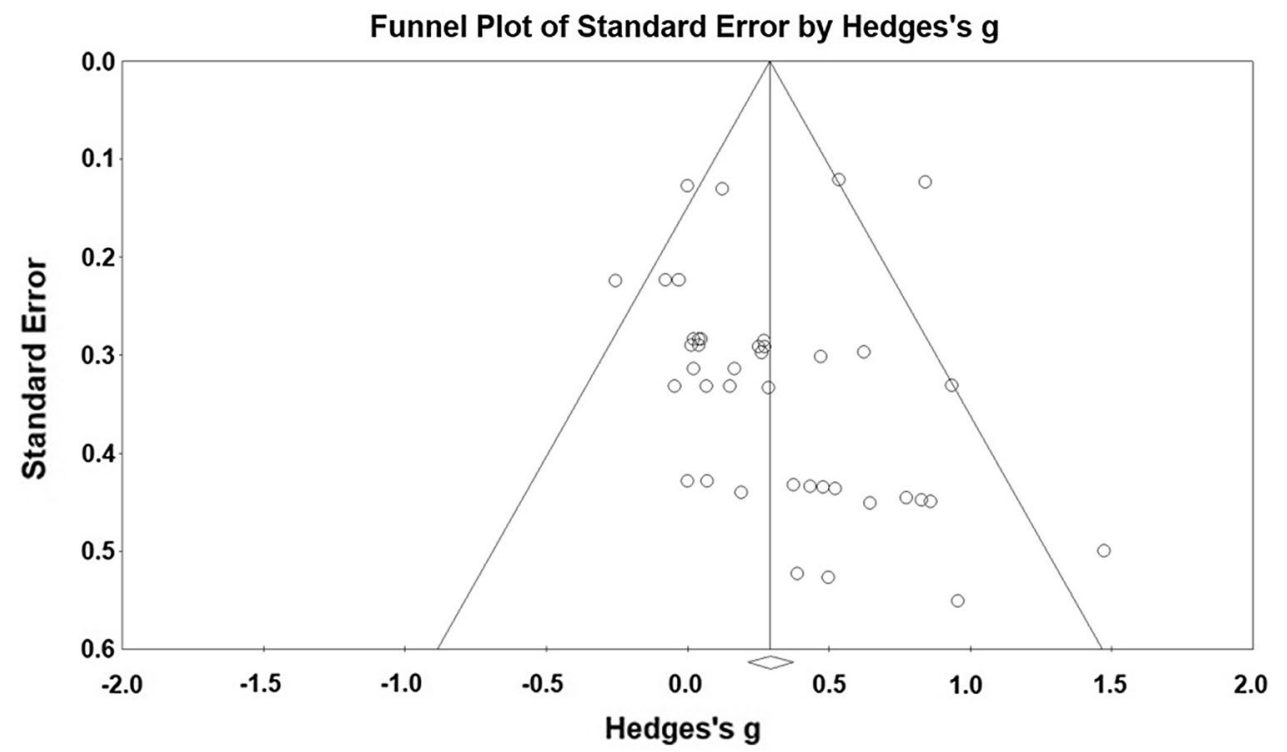

Fig. 3 Individual study and pooled results of MF training outcomes. $B W$ bodyweight, Trad traditional, Plyo plyometric, $C A$ combined activities. Letters $\mathrm{a}$ and $\mathrm{b}$ were used to separate studies investigating more than one type of resistance training

\section{Discussion}

To date, the literature has primarily focussed on the aerobic MVPA aspect of the PA guidelines, often overlooking MF [76]. Furthermore, adolescent boys are under-represented in the literature relative to girls [77]. This review builds upon the current literature by investigating the MF construct of PA. Our findings demonstrated that MF interventions were effective, which concurs with current literature, suggesting that adolescent boys may be receptive to MF interventions [78]. However, the small-to-moderate findings of this review should be interpreted with caution and considered in light of the high heterogeneity and a lack of specificity regarding the desired MF outcome in the studies. Moreover, the use of the term "strength training" within the literature is often misused, disregarding the independent nature of training adaptations to differing exercise modalities and overlooking the principle of specificity [79]. The concern of inappropriate inference to outcome measure has been recently raised [80] and the findings of this review suggest that there is also a lack of outcome measure specificity for MF and strength training in school-based studies. 
Fig. 4 Individual and pooled subgroup analyses of upper limb and lower limb MF outcomes. Studies with more than one outcome of MF are reported separately with the letter a allowing for separation between LL and UL outcomes. $L L$ lower limb; $U L$ upper limb

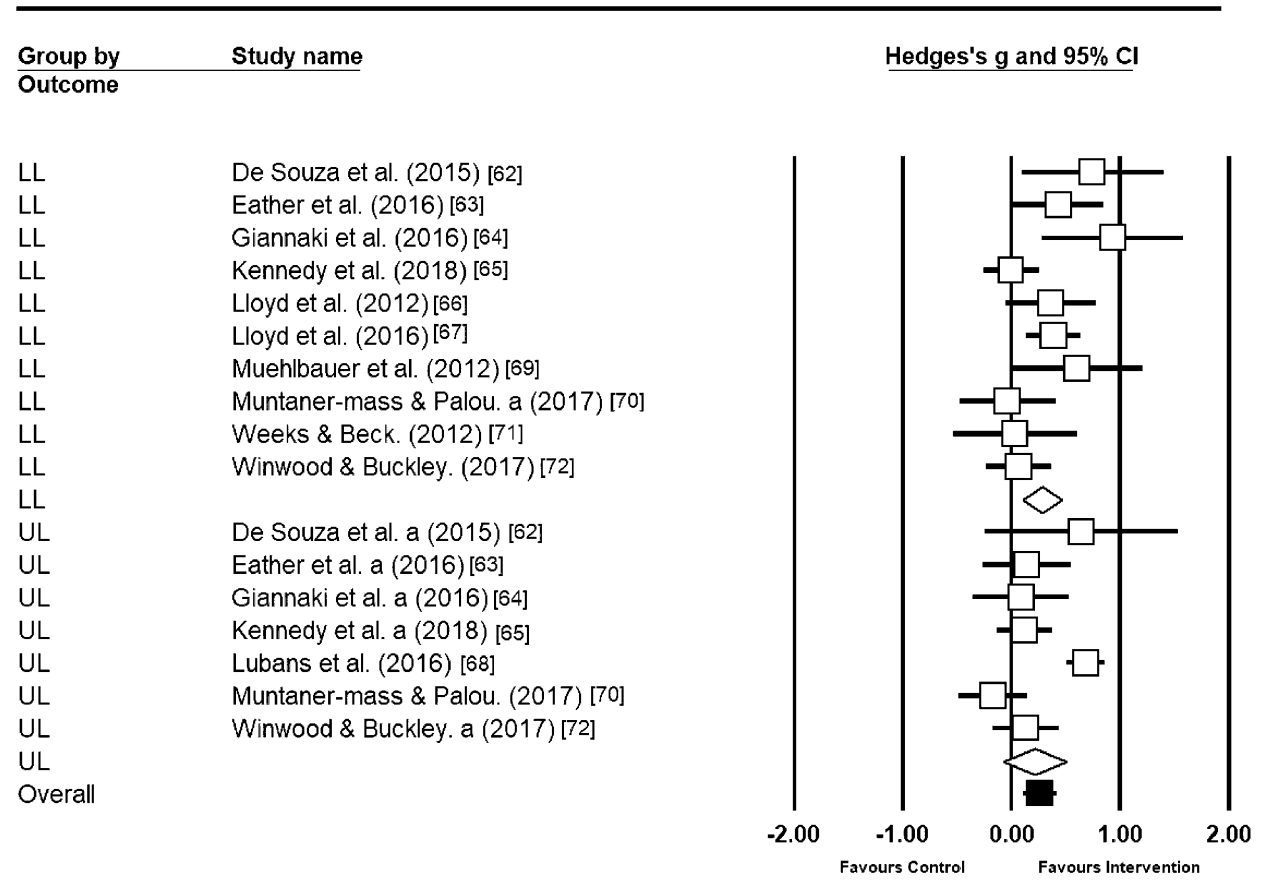

The literature suggests that MF interventions lasting 8-12 weeks are most effective in adolescent populations [8, $81,82]$. Seventy-two percent of studies investigating MF interventions met or exceeded this, suggesting that intervention duration may not have been long enough in over a quarter of studies to evoke an efficacious response. It is acknowledged that MF must adhere to underlying physiological characteristics that affect muscular strength to elicit an efficacious response and/ or adaptation [83]. Furthermore, the development of strength is underpinned by a combination of neural and morphological factors that may not be specifically catered for by conducting combined activities that involve high-intensity circuit-based interventions [83]. Adolescence provides an opportunity for neural and architectural adaptations in the development of strength due to increases in anabolic and hormonal concentrations [84]. However, 21 of the 43 data sets investigating MF utilised combined activities and may have overlooked the existing evidence-based methods that educe a more favourable response to the development of $\mathrm{MF}$, such as specific set and repetition schemes combined with appropriate rest periods. However, the practicalities, compliance, and pedagogical considerations associated with designing an MF program have not been explored in the literature, and may explain the lack of clarity on appropriate MF intervention design for a school-based setting. Moreover, the implementation of school-based RT may be impaired by some teachers reporting a lack of expertise/qualification and low confidence in the delivery of PE [85] which may be further exacerbated through the introduction of RT which currently resides outside of traditional PE [86].
Interestingly, plyometric RT demonstrated a statistically significant, homogeneous effect. Plyometric training has been evidenced to benefit peak bone mass in adolescent girls [87], and though evidence in boys is currently lacking, similar responses may be expected. However, only two studies adhered to appropriate plyometric training protocols, supporting the need for further quality research in this method of RT. Plyometric forms of training show promise and may provide a way to enhance muscle and bone strength. However, if such protocols are to be used within schools, appropriate training must be provided to ensure the safety and efficacy of this mode of RT. Moreover, individual variability in biological age, training age, skill, and coordination will dictate prescription of training frequency, intensity, velocity, and volume of plyometric RT [88]. The complexities associated with plyometric RT may explain the lack of research. Thus, consideration to pedagogy and practical application beyond the research in a school-based environment requires further investigation.

A key finding of this analysis was that traditional methods of MF were most effective. These are similar to those commonly practiced in commercial gymnasium environments that adolescents may encounter after leaving school. Thus, exposure to traditional RT may allow for preparation towards the transition into a popular form of PA conducted by adults. Recommendations for loading protocols can expect to see loads of 5-10\% added once the individual can comfortably perform 15 repetitions of a given movement with good form [89]. This method of adding load to progress the intensity of the RT may allow for greater perceived autonomy, whilst ensuring load increases are controlled through traditional 
machines and equipment allowing for smaller incremental increases when compared to bands or bodyweight. Moreover, allowing individuals to regulate the load progressions may enhance the intrinsic appeal [90]. Furthermore, the potential for enhancing physical literacy through neuromuscular adaptation indicative of RT may allow for previously disengaged adolescents to enhance their competence and participate in PA with greater intent and vigour. Adolescents that are overweight or obese may outperform their leaner peers when conducting the traditional forms of RT expressed in an in an absolute manner [51]. This may be due to their increased fat mass being indicative of a higher fat free mass, and thus, obese and overweight individuals may be able to lift or move more weight than leaner adolescents. Collectively, this greater involvement and ability to exercise competently alongside their peer group may allow for the relatedness component of self-determination theory (SDT) to be satisfied. Further research is warranted and should investigate SDT as a psychological construct to inform RT intervention design and content.

Subgroup analyses of muscle group was conducted to explore potential variance in MF outcomes, attributed to growth, maturation, and peak strength velocity occurring approximately 2 years after peak height velocity in adolescent boys [91, 92]. Evidence suggests that children and adolescents have a reduced ability to recruit type 2 muscle fibres, resulting in a lower voluntary muscle strength, speed, and power output [93-95]. Interventions conducted in the school environment may provide variance as to when students reach PHV and in turn, PSV. School-based interventions delivered to a broad range of youth should focus on developing muscle groups that may produce a homogeneous effect across a variety of ages, abilities, environments, and attitudes towards PA. This systematic review and metaanalysis demonstrated that lower limb MF outcomes $(n=27)$ had a homogeneous small-to-medium effect when compared to upper limb outcomes. This is irrespective of the potential for variance in ability, age, and attitude towards PA, and suggests that interventions targeting lower limb may be more effective than interventions designed to target upper limb. However, these results should be interpreted with caution as seven different measures to assess lower limb strength were used throughout the studies. Future research should standardise the use of lower limb strength measurements to assess and contextualise the efficacy of RT and its impact on lower limb development in the school environment. The findings of this review suggest that lower limb strength can be increased in a school-based setting across a broad spectrum of ages, abilities, and body types. Investing more time into the development of lower limb MF may support lowering the high percentage of lower limb injuries currently witnessed in active adolescent males [96], allowing those active individuals to continue PA within and beyond formal education. Furthermore, it has been suggested that the loss of muscle mass associated with the ageing process later in life may result in reductions in PA, with lower limb muscle groups being particularly susceptible to this phenomenon [97]. The findings of this review suggest that school-based interventions may contribute to homogeneous development of lower limb MF in adolescent males and contribute towards mitigating age-related declines through effective and early development of lower limb MF.

Methods of assessing upper limb strength $(n=14)$ were consistent across all seven studies. Press ups and grip strength featured in five and four of the studies, respectively, with one study assessing medicine ball throw. However, grip strength for upper limb assessment may not be the most reflective of those movements conducted during everyday life or as part of an exercise training regime [18, 98]. Recently, back leg and chest dynamometry has been validated in adolescents and may provide a cost effective, mobile, and simple tool to assess overall limb strength [99, 100]. To date, no school-based interventions investigating MF have utilised back leg and chest dynamometry as a measure to assess overall limb strength. Future research should consider the use of back leg and chest dynamometry to provide a measure of overall strength that may be more aligned to everyday life and as a marker of health [18, 99]. Upper limb MF outcomes did not provide a homogeneous outcome despite the consistency in assessment measures. This may be attributed to the variance in ages, both biologically and chronologically having an impact on force generation of the upper limb due to restriction in type 2 muscle fibre utilisation [101]. There may be a pedagogical concern when considering some of the functional shortcomings in adolescent boys, especially when attempting to design intervention and training protocols for this population group [96]. Although data are limited, it is suggested that upper limb RT may account for a larger proportion of injuries in early adolescence [96]. Further research is required to account for the heterogeneity in MF outcomes of the upper limb, and provide practitioners with appropriate, safe, and effective stimulus to enhance MF in adolescent males.

Only two studies objectively measured trunk strength. Trunk strength measures are simple to conduct and may inform the health of the lower back [102]. Although measures of trunk strength are simple to conduct in a field-based setting, researchers may be discouraged by the lengthy familiarisation process [103]. Researchers should explore methods that support a reduced familiarisation period or introduce familiarisation methods before intervention and data collection.

Reporting of the school-based MF interventions is sparse within the literature [104]. Furthermore, the utilisation of behavioural theory and socio-ecological models to underpin the delivery of MF interventions are not widely used. 
This may be due to recent work, suggesting that these models and constructs may not elicit a favourable outcome in the delivery of PA interventions investigating aerobic MVPA [105-108], resulting in a lack of willing to explore behavioural constructs when designing interventions. The school-based environment is unique in providing a largely mandatory setting to a broad range of youth [109]. Future intervention design may benefit from exploring enhanced, extended, and expanded opportunities (TEO) for youth PA and MF development in conjunction with complex behavioural theories [109] and avoid repeating the shortcomings evidenced in school-based aerobic MVPA intervention design [105-108]. TEO allows for a pragmatic approach to intervention design, expanding on PA opportunity by adding to the current PA opportunities, extending PA by adding additional time to current PA opportunities and, enhancing PA by augmenting existing PA opportunities [109]. Addressing both TEO and motivational psychological constructs may enhance the quality of the PA experience and positively impact intervention outcomes [109]. At an age where adolescent males may be preparing to leave the formal education environment, providing an opportunity to participate in RT may fulfil both a desire [110] and a need to explore a mode of PA that supports lifelong PA [111]. Future research should utilise TEO to allow both teachers and students to become familiar with the prescription of RT through the addition of its use within a school-based setting. This may help dispel some of the myths surrounding implementation (i.e., the need for specialist equipment and RT can damage growth) [112] and cultivate future intervention design.

Although RT in schools is still a developing concept, examples of periodized implementation have been reported when integrating RT [113]. As discussed, the correct implementation of an RT program is reliant upon accurate and appropriate testing to ensure that the practitioner can assign the correct volume and intensity to progress the adolescent [83]. Previously, testing protocols in the school environment have been greeted with trepidation from parents [114]. Traditionally fitness testing has been aerobically, or bodyweight centred, which may negatively impact physical self-concept in overweight and obese adolescents [114-116]. However, the nature of assessing MF can provide a way of overweight and obese adolescents to demonstrate their increased absolute strength when compared to their leaner peers [51]. Highlighting the areas in which adolescents excel physically may support positive relationships with PA, sport, and PE.

In addition to the testing considerations necessary for the implementation of RT interventions, the timing and period of delivery is equally as important $[8,81,82]$. The school environment lends itself well to the development of macrocycles that cover an academic year [113]. Furthermore, the structure of terms within the academic year could provide a way to develop detailed planning lasting between 2-6 weeks in the form of a mesocycle [117]. Consideration to time constraints placed upon the school should be taken into consideration when developing future interventions. Typically, exposure to PA is conducted within PE sessions lasting 45-60 min [118], allowing for a suitable amount of time to conduct effective RT in the school setting [119]. Overall, methods of constructing long-term planning are not only pragmatically appropriate to the school environment, but also widely recognised with RT literature, in both youth and adults [83]. Future research should consider the potential for the academic year to act as a construct for periodisation, whilst adhering to recognised protocols for RT to enhance specific MF adaptations. RT in schools should be approached with an informed appreciation for the nuances involved in program design, delivery, and a clear objective of the MF adaptation required. For delivery success at a larger scale, training must be provided to teachers and school coaches to confidently and effectively deliver RT.

\section{Strengths of this Review and Meta-analysis}

To the authors' knowledge this review is the first to address the efficacy of school-based PA interventions on MF outcomes in adolescent boys. This systematic review and metaanalysis are novel by way of addressing MF outcomes which are an element of youth PA guidelines. Further strengths were that the process to locate and extract all relevant data was rigorous and utilised an experienced librarian to ensure a comprehensive search strategy. Moreover, the grey literature search ensured that relevant non-peer-reviewed information was not missed.

\section{Limitations and Recommendations for Future Research}

There are limitations to this study that should be considered when interpreting the results. Although this review aimed to provide an international reference based upon the publication of the WHO PA guidelines [1], it should be noted that recommendations for RT were made in the 2008 American PA guidelines [120] and in earlier publications [121]. However, many countries are yet to develop their own PA policy and may utilise the WHO PA guidelines [1] as a global reference to inform their national PA guidelines and policy [54]. Furthermore, continuity of assessment method for MF interventions varied greatly, especially in the lower limb. The way in which training regimes were administered may also impact the outcome within the interventions, it 
is well understood that the end result of MF is determined by how the intervention is delivered and further research should seek to contextualise this to appropriately inform future practice [83]. Future research should investigate how differing MF delivery impacts the efficacy and outcome of the intervention.

Additionally, qualitative measures should be utilised to address the concerns of adolescent boys reported within the literature, with a third reporting a desire to enhance muscular aesthetics and another third reportedly wanting to become leaner $[122,123]$. Furthermore, it has been hypothesised that adolescent boys may be more inclined to participate in MF activities that are deemed more masculine [45]; this may have an impact on habitual PA. To date, the literature investigating the potential effect enhancing MF has on habitual PA has not been appropriately investigated and requires further work. Due to an insufficient amount of studies available reporting MF outcome aim (i.e., muscular endurance and power), analysis of specific adaptation outcomes could not be completed. Future research should be encouraged to provide an outcome measure such as increasing muscular endurance, power, or hypertrophy, so that future inferences and recommendations can be based upon the intervention outcome.

Future research should standardise MF assessment methods for use within adolescent population groups. Accurate measures of MF outcomes should be a documented within the literature to provide reliable measurement tools. Poor reliability may lead to erroneous conclusions about the MF parameter being measured. Studies investigating changes in MF should consider the whole intervention and how conflicting training modalities may impact MF outcomes. Finally, analysis of further moderators such as age (chronological and biological) and method of delivery (i.e., teacher or researcher delivered) was not possible due to insufficient detail contained within the literature. Future research should consider the impact of age and delivery method during interventions and report the methods within the study.

\section{Conclusions}

This systematic review and meta-analysis found a significant small effect for school-based MF interventions in adolescent boys. Efforts should be made to investigate the often overlooked MF element of the PA guidelines which promote and support physical and psychological health in youth. Traditional and plyometric methods of RT demonstrated the greatest effect when compared to other forms of RT, such as body weight movements, and require further research to draw more generalisable conclusions to inform long-term intervention design
Acknowledgements We would like to thank all authors who responded to our requests for additional information.

Author Contributions $\mathrm{AC}, \mathrm{SF}, \mathrm{MK}$, and RN all participated in the study design, protocol, and registration. $\mathrm{AC}$ and $\mathrm{RN}$ were responsible for selecting articles for inclusion and conducted the risk of bias assessment. AC and RN were responsible for data extraction. AC, SF, MK, and $\mathrm{RN}$ contributed to the data analysis. AC drafted the manuscript and all authors provided critical input and final approval.

Availability of data and materials After publication, all data necessary to understand and assess the conclusions of the manuscript are available to any reader of Sports Medicine.

\section{Compliance with Ethical Standards}

Funding No financial support was received for the conduct of this systematic review or preparation of this manuscript.

Conflict of interest The authors Ashley Cox, Stuart J. Fairclough, Maria-Christina Kosteli, and Robert J. Noonan declare that they have no competing interests.

Open Access This article is distributed under the terms of the Creative Commons Attribution 4.0 International License (http://creativeco mmons.org/licenses/by/4.0/), which permits unrestricted use, distribution, and reproduction in any medium, provided you give appropriate credit to the original author(s) and the source, provide a link to the Creative Commons license, and indicate if changes were made.

\section{References}

1. World Health Organization. Global Recommendations on Physical Activity for Health. Geneva: WHO Press; 2010.

2. Chief Medical Office. UK Physical Activity Guidelines; 2011. https://www.gov.uk/government/publications/uk-physical-activ ity-guidelines.

3. Physical Activity Guidelines for Americans, 2nd Edition, Executive Summary. health.gov. Office of Disease Prevention and Health Promotion. https://health.gov/paguidelines/second-editi on/pdf/PAG_ExecutiveSummary.pdf.

4. Health Council of the Netherlands. Physical activity guidelines 2017. The Hague: Health Council of the Netherlands, 2017;08.

5. Poitras VJ, Gray CE, Borghese MM, Carson V, Chaput J-P, Janssen I, et al. Systematic review of the relationships between objectively measured physical activity and health indicators in schoolaged children and youth. Appl Physiol Nutr Metab. 2016;41.

6. Collins H, Fawkner S, Booth JN, Duncan A. The effect of resistance training interventions on weight status in youth: a metaanalysis. Sports Med. 2018;41.

7. Bea JW, Blew RM, Howe C, Hetherington-Rauth M, Going SB. Resistance training effects on metabolic function among youth: a systematic review. Pediatr Exerc Sci. 2017;29.

8. Drenowatz C, Greier K. Resistance training in youth-benefits and characteristics. J Biomed. 2018;3.

9. Padilla-Moledo C, Ruiz JR, Ortega FB, Mora J, Castro-Piñero J. Associations of muscular fitness with psychological positive health, health complaints, and health risk behaviours in Spanish children and adolescents. J Strength Cond Res. 2012. 
10. Benson, AC, Torode ME, Fiatarone Singh MA. Effects of resistance training on metabolic fitness in children and adolescents: a systematic review. Obes Rev. 2008.

11. Health Behaviour in School-aged Children (HBSC) survey. Physical activity in adolescents. 2014.

12. Sandercock GRH, Cohen DD. Temporal trends in muscular fitness of English 10-year-olds 1998-2014: an allometric approach. J Sci Med Sport. 2018.

13. Tremblay MS, Shields M, Laviolette M, Craig CL, Janssen I, Gorber SC. Fitness of Canadian children and youth: results from the 2007-2009 Canadian Health Measures Survey. Health Rep. 2010;21:7-20.

14. Moliner-Urdiales D, Ruiz JR, Ortega FB, Jimenez Pavo D, Vicente-Rodriguez G, Rey-Lopez JP. Secular trends in healthrelated physical fitness in Spanish adolescents: the AVENA and HELENA Studies. J Sci Med Sport. 2010;13:584-8.

15. Albon HM, Hamlin MJ, Ross JJ. Secular trends and distributional changes in health and fitness performance variables of 10-14-year-old children in New Zealand between 1991 and 2003. Br J Sports Med. 2010;44:263-9.

16. Magnussen CG, Schmidt MD, Dwyer T. Muscular fitness and clustered cardiovascular disease risk in Australian youth. Eur J Appl Physiol. 2012;112.

17. Moliner-Urdiales D, Ruiz JR, Vicente-Rodriguez G, et al. Associations of muscular and cardiorespiratory fitness with total and central body fat in adolescents: the HELENA study. Br J Sports Med. 2011;45.

18. Smith JJ, Eather N, Morgan PJ, Plotnikoff RC, Faigenbaum AD, Lubans DR. The health benefits of muscular fitness for children and adolescents: a systematic review and meta-analysis. Sports Med. 2014;44:1209-23.

19. Artero EG, Ruiz JR, Ortega FB. Muscular and cardiorespiratory fitness are independently associated with metabolic risk in adolescents: the HELENA study. Paediatric Diabetes. 2011;12.

20. Cohen DD, Gomez-Arbelaez D, Camacho. Low muscle strength is associated with metabolic risk factors in Colombian children: the ACFIES study. PLoS One. 2014;9.

21. Ortega FB, Ruiz JR, Castillo MJ. Physical fitness in childhood and adolescence: a powerful marker of health. Int J Obes. 2008;32.

22. Fedewa AL., Ahn S. The effects of physical activity and physical fitness on children's achievement and cognitive outcomes: a meta-analysis. Res Q Exerc Sport. 2011;82.

23. Singh A, Uijtdewilligen L, Twisk JWR. Physical activity and performance at school: a systematic review of the literature including a methodological quality assessment. Arch Pediatr Adolesc Med. 2012;166.

24. García-Hermoso A, Ramírez-Campillo R, Izquierdo M. Is muscular fitness associated with future health benefits in children and adolescents? a systematic review and meta-analysis of longitudinal studies. Sports Med. 2019.

25. Faigenbaum AD, Kraemer WJ, Blimkie CJ, Jeffreys I, Micheli LJ, Nitka M,Rowland TW. Youth resistance training: updated position statement paper from the national strength and conditioning association. J Strength Cond Res. 2009.

26. Stratton G, Jones M, Fox KR, et al. BASES position statement on guidelines for resistance exercise in young people. J Sports Sci. 2004;22.

27. Pinedo-Villanueva R, Westbury LD, Syddall HE, Sanchez-Santos MT, Dennison, EM, Robinson SM, Cooper C. Health care costs associated with muscle weakness: a UK population-based estimate. Calcif Tissue Int. 2018.

28. Janssen I, Shepard DS, Katzmarzyk PT, Roubenoff R. The healthcare costs of sarcopenia in the United States. J Am Geriatr. 2004;52.
29. Reginster JY, Cooper C, Rizzoli R, Kanis JA, Appelboom G, Baut-mans I. Recommendations for the conduct of clinical trials for drugs to treat or prevent sarcopenia. Aging Clin Exp Res. 2016;28.

30. Kriemler S, Meyer U, Martin E, van Sluijs EMF, Andersen LB, Martin BW. Effect of school-based interventions on physical activity and fitness in children and adolescents: a review of reviews and systematic update. Br J Sports Med. 2011;45.

31. Fairclough SJ, Ridgers ND, Welk G. Correlates of children's moderate and vigorous physical activity during weekdays and weekends. J Phys Act Health. 2012;9.

32. Love R, Adams J, Van Sluijs EMF. Are school-based physical activity interventions effective and equitable? A meta-analysis of cluster randomized controlled trials with accelerometer-assessed activity. Obes Rev. 2019.

33. Physical Activity Review Group. Five year review of 'Let's make Scotland more active: a strategy for physical activity': update statement from the review group. Edinburgh: NHS Health Scotland; 2010.

34. Scotland Sport. Increasing demand for sport and physical activity for adolescent girls in Scotland: Exploring issues and suggesting solutions. Edinburgh: Sport Scotland; 2006.

35. Physical Activity and Health Alliance. Physical activity and adolescent girls-Knowledge Exchange Network. 2009.

36. Hallal PC, Andersen LB, Bull FC, et al. Lancet Physical Activity Series Working Group. Global physical activity levels: surveillance progress, pitfalls, and prospects. Lancet. 2012;380.

37. Flegal KM. The obesity epidemic in children and adults: current evidence and research issues. Med Sci Sports Exerc. 1999;31.

38. Fox K. Tackling obesity in children through physical activity: a perspective from the United Kingdom. Quest. 2004;56.

39. Centres for Disease Control and Prevention. Childhood obesity facts. 2013.

40. World Health Organization. Obesity: preventing and managing the global epidemic. 2004;894.

41. Public Health England. Fingertips Data. Local Authority Health Profiles. Excess weights in adults; 2017. https://fingertips.phe. org.uk/profile/health-profiles/data\#page/7/gid/1938132694/ pat/15/par/E92000001/ati/6/are/E12000002/iid/93088/age/168/ sex/4.

42. Australian Institute of Health and Welfare. A picture of overweight and obesity in Australia 2017. Canberra: AIHW; 2017.

43. Abarca-GÃ L, Abdeen ZA, Abdul Hamid Z, Abu-Rmeileh NM, Acosta-Cazares B, Acuin C. NCD Risk Factor Collaboration. Worldwide trends in body-mass index, underweight, overweight, and obesity from 1975 to 2016: a pooled analysis of 2416 population-based measurement studies in 128.9 million children, adolescents, and adults. Lancet. 2017.

44. Jachyra P, Gibson BE. LIFE AND HEALTH CARE TRANSITIONS: boys, transitions, and physical inactivity: exploring the socio-behavioural mediators of participation. Physiother Can. 2016;68.

45. Lubans DR, Cliff DP. Muscular fitness, body composition and physical self-perception in adolescents. J Sci Med Sport. 2011;14.

46. Gray JJ, Ginsberg RL. Muscle dissatisfaction: an overview of psychological and cultural research and theory. Muscular ideal: psychological social, and medical perspectives. Washington, DC; 2007.

47. Faigenbaum AD, Myer GD. Paediatric resistance training: benefits, concerns, and program design considerations. Curr Sports Med Rep. 2010;9.

48. De Ste Croix MBA, Deighan MA, Armstrong N. Assessment and interpretation of isokinetic strength during growth and maturation. Sports Med. 2003;33:727-43. 
49. De Ste Croix MBA. Advances in paediatric strength testing: changing our perspective on age and sex associated differences in muscle strength. J Sports Sci Med. 2007;6:292-304.

50. Wood LE, Dixon S, Grant C, Armstrong N. Elbow flexor strength, muscle size, and moment arms in prepubertal boys and girls. Pediatr Exerc Sci. 2006;18:457-69.

51. Ten Hoor GA, Kok G, Rutten GM, et al. The Dutch 'Focus on Strength' intervention study protocol: programme design and production, implementation and evaluation plan. BMC Public Health. 2016;16.

52. Metcalf B, Henley W, Wilkin T. Republished research: Effectiveness of intervention on physical activity of children: systematic review and meta-analysis of controlled trials with objectively measured outcomes. Br J Sports Med. 2013;47.

53. Haddaway NR, Collins AM, Coughlin D, Kirk S. The role of google scholar in evidence reviews and its applicability to grey literature searching. PLoS One. 2015;10.

54. Sallis JF, Bull F, Guthold R, Heath GW, Inoue S, Kelly P, Oyeyemi AL, Perez LG, Richards J, Hallal PC, Lancet Physical Activity Series 2 Executive Committee. Progress in physical activity over the Olympic quadrennium. Lancet. 2016;388:1325-36.

55. Sikich NNI. Quality Control Tool for Screening Titles and Abstracts by second Reviewer. J Biom Biostat. 2015.

56. Pluye P, Gagnon MP, Griffiths F, Johnson-Lafleur J. A scoring system for appraising mixed methods research, and concomitantly appraising qualitative, quantitative and mixed methods primary studies in mixed studies reviews. Int J Nurs Stud. 2009;46.

57. Morton KL, Atkin AJ, Corder K, Suhrcke M, van Sluijs EMF. The school environment and adolescent physical activity and sedentary behaviour: a mixed-studies systematic review. Obes Rev. 2015;17.

58. Clark TS, Linzer DA, Beardsley K., Esarey J, Gelman A, Imai $\mathrm{K}$, et al. Should I use fixed or random effects? Political Sci Res Methods. 2015;3.

59. Cohen J. Statistical power analysis for the behavioural sciences. 2nd ed. Hillsdale: Lawrence Earlbaum Associates; 1988.

60. Higgins JP, Thompson SG, Deeks JJ. Measuring inconsistency in meta- analyses. Br Med J. 2003;327.

61. Egger M, Davey Smith G, Schneider M, et al. Bias in metaanalysis detected by a simple, graphical test. Br Med J. 1997;315.

62. de Souza S, Oliveira D, Pereira T, et al. Does a CalisthenicsBased Exercise Program Applied in School Improve Morpho functional Parameters in Youth? J Exerc Physiol Online. 2015;18.

63. Eather N, Morgan PJ, Lubans DR. Improving health-related fitness in adolescents: the CrossFit Teens ${ }^{\mathrm{TM}}$ randomised controlled trial. J Sports Sci. 2016;34.

64. Giannaki CD, Aphamis G, Tsouloupas CN, Ioannou Y, Hadjicharalambous M. An eight week school-based intervention with circuit training improves physical fitness and reduces body fat in male adolescents. J Sports Med Phys Fitness. 2016;56.

65. Kennedy SG, Smith JJ, Morgan PJ, Peralta LR, Hilland, TA, Eather, N, Lubans DR, et al. Implementing resistance training in secondary schools. Med Sci Sports Exerc. 2017.

66. Lloyd RS, Oliver JL, Hughes MG, Williams CA. The effects of 4-weeks of plyometric training on reactive strength index and leg stiffness in male youths. J Strength Cond Res. 2012;26.

67. Lloyd RS, Radnor JM, De Ste Croix MB, Cronin JB, Oliver JL. Changes in sprint and jump performances after traditional, plyometric, and combined resistance training in male youth pre- and post-peak height velocity. J Strength Cond Res. 2016;30.

68. Lubans DR, Smith JJ, Plotnikoff RC, Dally KA, Okely AD, Salmon J, Morgan PJ. Assessing the sustained impact of a school-based obesity prevention program for adolescent boys: the ATLAS cluster randomized controlled trial. Int J Behav Nutr Phys Act. 2016;13.
69. Muehlbauer T, Gollhofer A, Granacher U. Sex-related effects in strength training during adolescence: a pilot study. Percept Mot Skills. 2012;115.

70. Muntaner-Mas, Palou A. Effects of high intensity interval training (HIIT) intervention amongst school adolescents. J Phys Ed Health. 2017;6.

71. Weeks BK, Beck BR. Brief jumping bouts improve lean mass in boys. Pediatr Obes. 2012;7.

72. Winwood PW, Buckley JJ. Short-term effects of resistance training modalities on performance measures in male adolescents. $\mathbf{J}$ Strength Cond Res. 2019;33.

73. Nicol C, Avela J, Komi P. The stretch-shortening cycle: a model to study naturally occurring neuromuscular fatigue. Sports Med. 2006;36:977-99.

74. Flanagan EP, Comyns TM. The use of contact time and the reactive strength index to optimize fast stretch-shortening cycle training. Strength Cond J. 2008;30:32-8.

75. Channell BT, Barfield JP. Effect of olympic and traditional resistance training on vertical jump improvement in high school boys. J Strength Cond Res. 2008;22.

76. Eliakim A, Falk B, Armstrong N, Baptista F, Behm DG, Dror, N, et al. Aerobic exercise and training during youth. Pediatr Exerc Sci. 2019;31.

77. Cooper AR, Goodman A, Page AS, Sherar LB, Esliger DW, van Sluijs EM, Ekelund U. Objectively measured physical activity and sedentary time in youth: The International children's accelerometry database (ICAD). Int J Behav Nutr Phys Act. 2015;12.

78. Gray JJ, Ginsberg RL. Muscle dissatisfaction: an overview of psychological and cultural research and theory. Muscular ideal: psychological social, and medical perspectives. 2007.

79. Gamble P. Implications and applications of training specificity for coaches and athletes. Strength Cond J. 2006;28:54-8.

80. Moran J, Sandercock GRH, Ramírez-Campillo R, Meylan C, Collison J, Parry DA. A meta-analysis of maturation-related variation in adolescent boy athletes' adaptations to short-term resistance training. J Sports Sci. 2017;35:1041-51.

81. Behringer M, Vom Heede A, Yue Z, Mester J. Effects of resistance training in children and adolescents: a meta-analysis. Paediatrics. 2010;126.

82. Faigenbaum AD, Kraemer WJ, Blimkie CJ, Jeffreys I, Micheli LJ, Nitka M, Rowland TW. Youth resistance training: updated position statement paper from the national strength and conditioning association. J Strength Cond Res. 2009;23.

83. Suchomel TJ, Nimphius S, Bellon CR, Stone MH. The Importance of Muscular Strength: Training Considerations. Sports Med. 2018;48.

84. Moran J, Sandercock GRH, Ramírez-Campillo R, Meylan C, Collison J, Parry DA. A meta-analysis of maturation-related variation in adolescent boy athletes' adaptations to short-term resistance training. J Sports Sci. 2017;35.

85. Nathan N, Elton B, Babic M, McCarthy N, Sutherland R, Presseau J, Seward K, Hodder R, Booth D, Yoong SL. Barriers and facilitators to the implementation of physical activity policies in schools: a systematic review. Prev Med. 2018;107:43-53.

86. Kennedy SG, Peralta LR, Lubans DR, Foweather L, Smith JJ. Implementing a school-based physical activity program: process evaluation and impact on teachers' confidence, perceived barriers and self-perceptions. Phys Educ Sport Pedagogy. 2019;24:233-48.

87. Witzke KA, Snow CM. Effects of plyometric jump training on bone mass in adolescent girls. Med Sci Sports Exerc. 2000;32.

88. Lloyd RS, Oliver JL. The Youth Physical Development Model: a new approach to long term athletic development. Strength Cond J. 2012;34. 
89. Faigenbaum A, Kraemer W, Blimkie C, Jeffreys I, Micheli L, Nitka M, Rowland T. Youth resistance training: updated position statement paper from the National Strength and Conditioning Association. J Strength Cond Res. 2009;23.

90. Halperin I, Wulf G, Vigotsky AD, Schoenfeld BJ, Behm DG. Autonomy. Strength Cond J. 2018.

91. Rasmussen B, Kalusen K, Jespersen B, Jensen K. A longitudinal study of development in growth and maturation of 10- to 15-year old girls and boys. Children and exercise XIII. Champaign: Human Kinetics; 1990. p. 103-11.

92. Carvalho HM, Coelho-e-Silva M, Valente-dos-Santos J, Gonçalves RS, Philippaerts R, Malina R. Scaling lower-limb isokinetic strength for biological maturation and body size in adolescent basketball players. Eur J Appl Physiol. 2012;112:2881-9.

93. Cohen R, Mitchell C, Dotan R, Gabriel D, Klentrou P, Falk B. Do neuromuscular adaptations occur in endurance-trained boys and men? Appl Physiol Nutr Metab. 2010;35:471-9.

94. Dotan R, Mitchell C, Cohen R, Klentrou P, Gabriel D, Falk B. Child-adult differences in muscle activation-a review. Pediatr Exerc Sci. 2012;24:2-21.

95. Falk B, Brunton L, Dotan R, Usselman C, Klentrou P, Gabriel D. Muscle strength and contractile kinetics of isometric elbow flexion in girls and women. Pediatr Exerc Sci. 2009;21:354-64.

96. Faigenbaum AD, Myer GD. Resistance training among young athletes: safety, efficacy and injury prevention effects. Br J Sports Med. 2010

97. Candow DG, Chilibeck PD. Differences in size, strength, and power of upper and lower body muscle groups in young and older men. J Gerontol A Biol Sci Med Sci. 2005;60:148-56.

98. Abernethy P, Wilson G, Logan P. Strength and power assessment. Issues, controversies and challenges. Sports Med. 1995;19.

99. Ten Hoor GA, Musch K, Meijer K, Plasqui G. Test-retest reproducibility and validity of the back-leg-chest strength measurements. Isokinet Exerc Sci. 2016.

100. Smith JJ, Eather N, Weaver RG, Riley N, Beets MW, Lubans DR. Behavioural correlates of muscular fitness in children and adolescents: a systematic review. Sports Med. 2019;49:887-904.

101. Dotan R, Mitchell C, Cohen R, Gabriel D, Klentrou P, Falk B. Child-adult differences in the kinetics of torque development. $\mathrm{J}$ Sports Sci. 2013;31:945-53.

102. Moya-Ramón M, Juan-Recio C, Lopez-Plaza D, Vera-Garcia FJ. Dynamic trunk muscle endurance profile in adolescents aged 14-18: normative values for age and gender differences. J Back Musculoskelet Rehabil. 2018.

103. Brotons-Gil E, Garcia-Vaquero MP, Peco-Gonzalez N, VeraGarcia FJ. Flexion-rotation trunk test to assess abdominal muscle endurance: reliability, learning effect, and sex differences. J Strength Cond Res. 2013;27.

104. Janssen I, LeBlanc AG. Systematic review of the health benefits of physical activity and fitness in school-aged children and youth. Int J Behav Nutr Phys Act. 2010;7:40.

105. Tymms PB, Curtis SE, Routen AC, Thomson KH, Bolden DS, Bock S, Dunn CE, Cooper AR, Elliott JG, Moore HJ. Clustered randomised controlled trial of two education interventions designed to increase physical activity and well-being of secondary school students: the MOVE Project. BMJ Open. 2016.

106. Kipping RR, Howe LD, Jago R, Campbell R, Wells S, Chittleborough CR, Mytton J, Noble SM, Peters TJ, Lawlor DA. Effect of intervention aimed at increasing physical activity, reducing sedentary behaviour, and increasing fruit and vegetable consumption in children: active for Life Year 5 (AFLY5) school based cluster randomised controlled trial. BMJ. 2014.

107. Van Sluijs EM, Kriemler S, McMinn AM. The effect of community and family interventions on young people's physical activity levels: a review of reviews and updated systematic review. Br J Sports Med. 2011;45:914-22.

108. van Sluijs EM, McMinn AM, Griffin SJ. Effectiveness of interventions to promote physical activity in children and adolescents: systematic review of controlled trials. BMJ. 2007;335:703.

109. Beets MW, Okely A, Glenn Weaver R, Webster C, Lubans D, Brusseau T. The theory of expanded, extended, and enhanced opportunities for youth physical activity promotion. Int J Behav Nutr Phys Act. 2016;13.

110. Corder K, Atkin AJ, Ekelund U, van Sluijs EM. What do adolescents want in order to become more active? BMC Public Health 2013;13:718-27.

111. Hulteen RM, Smith JJ, Morgan PJ, et al. Global participation in sport and leisure-time physical activities: a systematic review and meta-analysis. Prev Med. 2017;95:14-25.

112. Steele J, Fisher J, Skivington M. A higher effort-based paradigm in physical activity and exercise for public health: making the case for a greater emphasis on resistance training. BMC Public Health. 2017;17:300.

113. Pichardo AW, Oliver JL, Harrison CB, Maulder PS, Lloyd RS. Integrating resistance training into high school curriculum. Strength Cond J. 2015;41:39-50.

114. Cohen DD, Voss C, Sandercock GRH. Fitness testing for children: let's mount the zebra! J Phys Act Health. 2015;12:597-603.

115. Martin SB, Ede A, Morrow JR, Jackson AW. State-wide physical fitness testing: perspectives from the gym. Res Q Exerc Sport. 2010;81:S31-41

116. Zhu W, Welk GJ, Meredith MD, Boiarskaia EA. A survey of physical education programs and policies in Texas schools. Res Q Exerc Sport. 2010;81:S42-52.

117. Haff GG, Haff E. Training integration and periodization. In: Hoffman J, editor. NSCA's guide to program design. Champaign: Human Kinetics; 2012. p. 209-54.

118. Duehring MD, Feldmann CR, Ebben WP. Strength and conditioning practices of United States high school strength and conditioning coaches. J Strength Cond Res. 2009;23:2188-203.

119. Lloyd RS, Faigenbaum AD, Stone MH, Oliver JL, Jeffreys I, Moody JA, Brewer C, Pierce KC, McCambridge TM, Howard R, Herrington L, Hainline B, Micheli LJ, Jaques R, Kraemer WJ, McBride MG, Best TM, Chu DA, Alvar BA, Myer GD. Position statement on youth resistance training: the 2014 International Consensus. Br J Sports Med. 2014;48:498-505.

120. Health Education Authority. Young and active?. London: H.E.A; 1998

121. US Department of Health and Human Services. Physical activity guidelines for Americans: be active, healthy and happy, Washington DC; 2008.

122. Furnham A, Calman A. Eating disturbance, self-esteem, reasons for exercising and body weight dissatisfaction in adolescent males. Eur Eat Disord Rev. 1998;6.

123. Ricciardelli LA, McCabe MP. A longitudinal analysis of the role of biopsychosocial factors in predicting body change strategies among adolescent boys. Sex Roles. 2003;48. 